\title{
Bridging Gaps in Imaging by Applying EM Tomography and Serial Block Face SEM, including a New Genetically Encoded Tag for Correlated Light and 3D Electron Microscopy of Intact Cells, Tissues and Organisms: Integrating the Resulting Correlated Image Data Using the Whole Brain Catalog
}

\author{
M. H. Ellisman*, X. Shu^, V. Lev-Ram^^, T. J. Deerinck*, R. Y. Tsien*^, S. P. Lamont*, J. \\ Martinez*, M. Berlanga*, E. Bushong*, M. E. Martone*, and S. Larson* \\ *University of California, San Diego, National Center for Microscopy and Imaging Research, \\ Center for Research in Biological Systems; ^Howard Hughes Medical Institute and Department \\ of Pharmacology, University of California at San Diego, La Jolla, CA 92093
}

A grand goal in neuroscience research is to understand how the interplay of structural, chemical and electrical signals in and between cells of nervous tissue gives rise to behavior. We are rapidly approaching this horizon as neuroscientists make use of an increasingly powerful arsenal of tools and technologies for obtaining data, from the level of molecules to nervous systems, and engage in the arduous and challenging process of adapting and assembling neuroscience data at all scales of resolution and across disciplines into computerized databases. This talk will highlight projects where development and application of new contrasting methods and imaging tools have allowed us to see otherwise hidden relationships between cellular, subcellular and molecular constituents of nervous systems. New chemistries for carrying out correlated light and electron microscopy will be described, as well as recent advances in large-scale high-resolution $3 \mathrm{D}$ reconstruction with TEM and SEM based methods. The Whole Brain Catalog (WBC), a Google Earth-like open-source virtual model of the mouse brain, will also be described. The WBC is as an example of an informatics framework and web-based tool whose purpose is partly to facilitate integration of 3D image data from multiple microscopy methods and to enable the linking of information derived from other analytical approaches to imaging data shared in the publically accessible catalog.

A New Genetically Encoded Tag for Correlated Light and Electron Microscopy: We have developed a small flavoprotein engineered to photogenerate singlet oxygen that efficiently serves as a genetically encoded tag to visualize specific proteins within intact cells, tissues, and organisms by optical and electron microscopy. While electron microscopy (EM) achieves the highest spatial resolution in protein localization, specific protein EM labeling has lacked generally applicable genetically encoded tags for in situ visualization in cells and tissues. We recently introduced "miniSOG" (for mini Singlet Oxygen Generator), a fluorescent flavoprotein engineered from Arabidopsis phototropin 2. MiniSOG contains 106 amino acids, less than half the size of Green Fluorescent Protein. Illumination of miniSOG generates sufficient singlet oxygen to locally catalyze the polymerization of diaminobenzidine into an osmiophilic reaction product resolvable by EM. MiniSOG fusions have been made for many well characterized proteins localize correctly in cultured cells and intact organisms, enabling correlated fluorescence and EM from large volumes of tissue after strong aldehyde fixation, without the need for exogenous ligands, probes or destructive permeabilizing detergents. MiniSOG permits high quality ultrastructural preservation and 3-dimensional protein localization via electron 
tomography or serial section block face scanning electron microscopy. MiniSOG may do for EM what Green Fluorescent Protein did for fluorescence microscopy.

Use of the Whole Brain Catalog (WBC) for Synthesis of data from multiple light and electron microscopic methods to produce a multiscale atlas from molecular to tissue scales: Several of the large scale image data collection instruments at the National Center for Microscopy and Imaging Research (NCMIR) are now streaming multiscale data about mouse animal models of disease and normal mice into the WBC. These include wide field reconstructions of cells in the nervous system using ultra-wide field laser scanning light microscopy, serial block-face scanning electron microscopy, ultra-wide field electron microscopy and tomography for whole cell reconstructions. These tools are applied to biomedical research projects including those pertaining to models of Parkinson's disease, cancer and epilepsy as part of the collaborative project / driving biomedical research project portfolio of the NCMIR which shape technological research and development at the Center. Most notably, extremely large volumes of data are already being fed into the WBC using a new 3D EM technique being refined at NCMIR. This method combines the new high throughput scanning EM method, "serial blockface scanning electron microscopy (SBFSEM)", developed by Winfried Denk and coworkers in Heidelberg with NCMIR-developed methods for high contrast staining and molecule-specific marking to facilitate correlated light and electron microscopy.

This Web-based resource also incorporates the 3D geometry of the Allen Reference Atlas structures of the mouse brain, with a NCMIR research collaborators images taken from the Cell Centered DataBase (CCDB) and integrated with collections from GENSAT mice and example mouse models of Parkinson's disease. The brain models of the WBC can be easily rotated, panned and zoomed in real time allowing interactive exploration. Zooming into the brain reveals further detail such as 3D representations of neurons, 3D representations of cellular substructure and so forth, like supramolecular complexes found in EM tomograms of neuropil or deposited in the Protein Data Bank. Images of unlimited size can be warped and aligned to atlases; and images of unlimited size can be viewed. 